\section{Cureus}

\title{
Synchronous Presentation of a Cervical Spinal Schwannoma and Primary Progressive Multiple Sclerosis in a 65-year- old Man
}

Avital Perry, Pierce Peters, Christopher S. Graffeo, Lucas P. Carlstrom, William E. Krauss

1.

Corresponding author: Pierce Peters, peters.pierce@mayo.edu

\section{Abstract}

Schwannomas are common benign neoplasms of the myelinating cells surrounding peripheral nerve axons. Though uncommon, lesions arising in the cervical spine may produce radicular pain and myelopathic weakness. Multiple sclerosis (MS) is a common autoimmune disorder with the capacity to mimic nearly any neurologic disease, including spinal cord neoplasms. We report the third case of synchronously presenting primary progressive MS and spinal schwannoma.

A 65-year-old man presented with six months of progressive weakness and pain of the right shoulder, forearm, and hand. MRI demonstrated a contrast-enhancing transforaminal lesion at $\mathrm{C} 7$, most consistent with a benign nerve sheath tumor. Additional history disclosed several years of worsening fatigue, accompanied by bilateral weakness and lancinating leg pain. MRI of the neuraxis demonstrated abnormalities consistent with chronic demyelinating disease intracranially and within the spinal cord; cerebrospinal fluid (CSF) analysis revealed nine oligoclonal bands and an elevated IgG index, resulting in the diagnosis of MS. Given the symptomatic C7 lesion, the patient subsequently underwent right C6-C7 facetectomy, gross total resection of the tumor, and C6-T1 posterior instrumented fusion. Postoperatively, the patient rapidly recovered normal right upper extremity function, and pathology confirmed benign schwannoma.

Synchronously presenting co-morbid neurologic diagnoses are exceedingly rare. Nonetheless, the high incidence and protean nature of MS make it particularly susceptible to such confounding clinical cases. Correspondingly, MS should be considered when neurologic abnormalities are not compatible with a focal radiographic lesion, and the present report emphasizes the value of a good history and exam in unraveling similarly challenging cases.

Received 10/02/2018

Review began 10/12/2018 Review ended 02/27/2019 Published 03/04/2019

(C) Copyright 2019

Perry et al. This is an open access article distributed under the terms of the Creative Commons Attribution License CC-BY 3.0., which permits unrestricted use, distribution, and reproduction in any medium, provided the original author and source are credited.
Categories: Neurosurgery

Keywords: spinal schwannoma, multiple sclerosis, differential diagnosis, schwannomatosis

\section{Introduction}

Spinal schwannoma is the most common intra-dural extra-axial neoplasm, comprising nearly one-third of tumors found within that anatomic space, with an incidence of $0.3-0.5$ per 100,000 , and a peak distribution during the fifth decade of life [1-3]. Multiple sclerosis (MS) is a highly prevalent demyelinating disease, occurring at an incidence of two per 100,000, with a mean age of 30 and a 2.3:1 female predominance [4-5]. Considered one of the "great imitators," MS manifests with a wide swath of confounding symptoms, and has been mistaken for nearly

How to cite this article

Perry A, Peters P, Graffeo C S, et al. (March 04, 2019) Synchronous Presentation of a Cervical Spinal Schwannoma and Primary Progressive Multiple Sclerosis in a 65-year-old Man. Cureus 11(3): e4176. DOI $10.7759 /$ cureus. 4176 


\section{Cureus}

every other common neurologic diagnosis, including spinal tumors [6-7].

Although far from universally applicable, Dr. William Osler's repurposing of Occam's Razorthat a single, unifying diagnosis should be sought to explain all of a given patient's symptomsremains salient, and in many cases provides a helpful tool for scrutinizing the differential and eliminating diagnoses that are simultaneous unlikely and supported [8]. Notwithstanding, as Dr. John Hickam proposed in his counterpoint to Osler's Rule, Hickam's Dictum, there are numerous circumstances, both pedestrian and rarified, that defy simplicity, and that practical, clinical evidence should universally trump theoretical postulates, allowing the patient to have "as many diagnoses as he damn well pleases" [9]. In the spirit of this dialogue, we present a rare case of synchronously presenting spinal schwannoma and multiple sclerosis-only the third such patient-reported, and an important illustration of the principles of attentive diagnosis with a detailed history and physical exam, resistance of radiographic search satisfaction, and openness to the unexpected, particularly where the clinical evidence flies in the face of Occam's Razor.

\section{Case Presentation}

A 65-year-old man presented with six months of progressive weakness and pain of the right shoulder, forearm, and hand. He was evaluated by his local neurologist and neurosurgeon, and an MRI was acquired and demonstrated findings consistent with a C7 nerve sheath tumor (Figure 1). Electromyogram (EMG) confirmed right C7 radiculopathy, and the patient was referred to our institution for neurosurgical treatment. Although congruent findings were noted on MRI and EMG, additional history was obtained that revealed highly concerning findings inconsistent with a cervical nerve root schwannoma. The patient had several years of progressively worsening fatigue with activity, bilateral leg weakness, and shooting pain, as well as bladder urgency without incontinence. Physical examination revealed right C7 weakness, marked gait unsteadiness, and positive Babinski sign on the left.

A

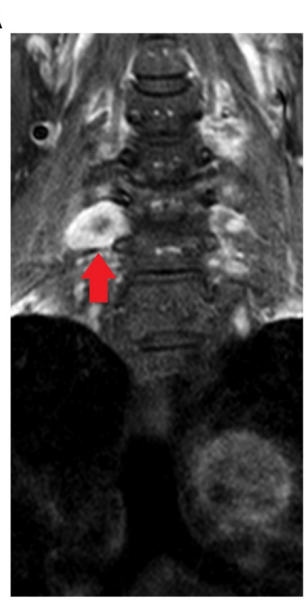

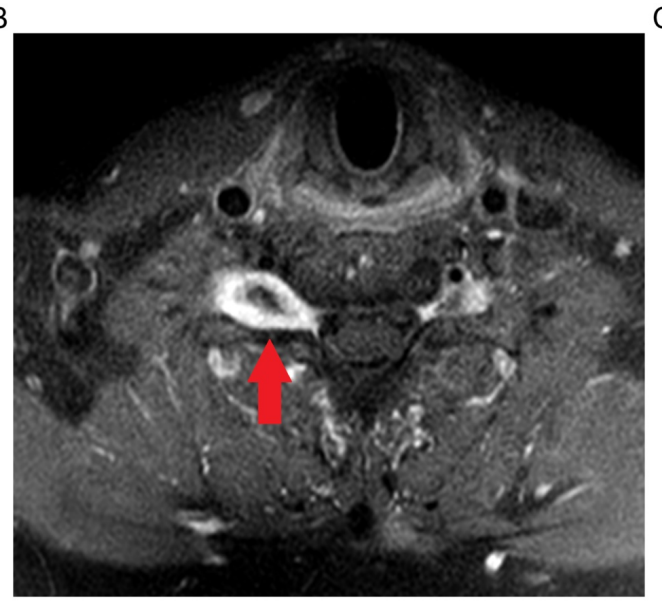

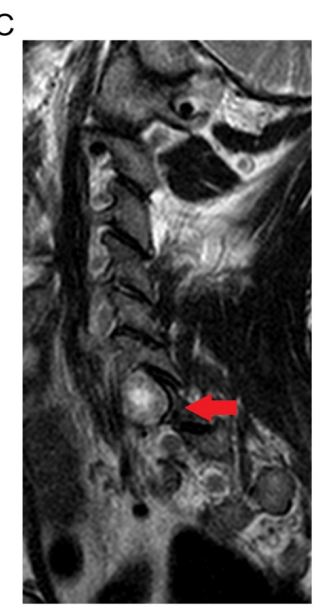

FIGURE 1: Gadolinium-enhanced T1-weighted coronal (A), axial (B), and T2-weighted sagittal (C) MRI signal highlighting a centrally cystic/necrotic nerve sheath tumor at the level of $\mathrm{C7}$ (arrows).

These new findings prompted MRI of the total neuraxis, which reproduced the right C7 mass, while also identifying subtle, intrinsic T2 signal abnormalities of the thoracic spinal cord 


\section{Cureus}

(Figure 2), and multiple periventricular T2 signal abnormalities (Figure 3), collectively raising significant concern for chronic demyelinating disease. Cerebrospinal fluid (CSF) analysis identified had nine oligoclonal bands with an elevated IgG index, and the diagnosis of primary progressive MS was made. Of note, the patient was assessed for benign prostatic hyperplasia, and it was thought unlikely to be responsible for his presenting urinary symptoms.

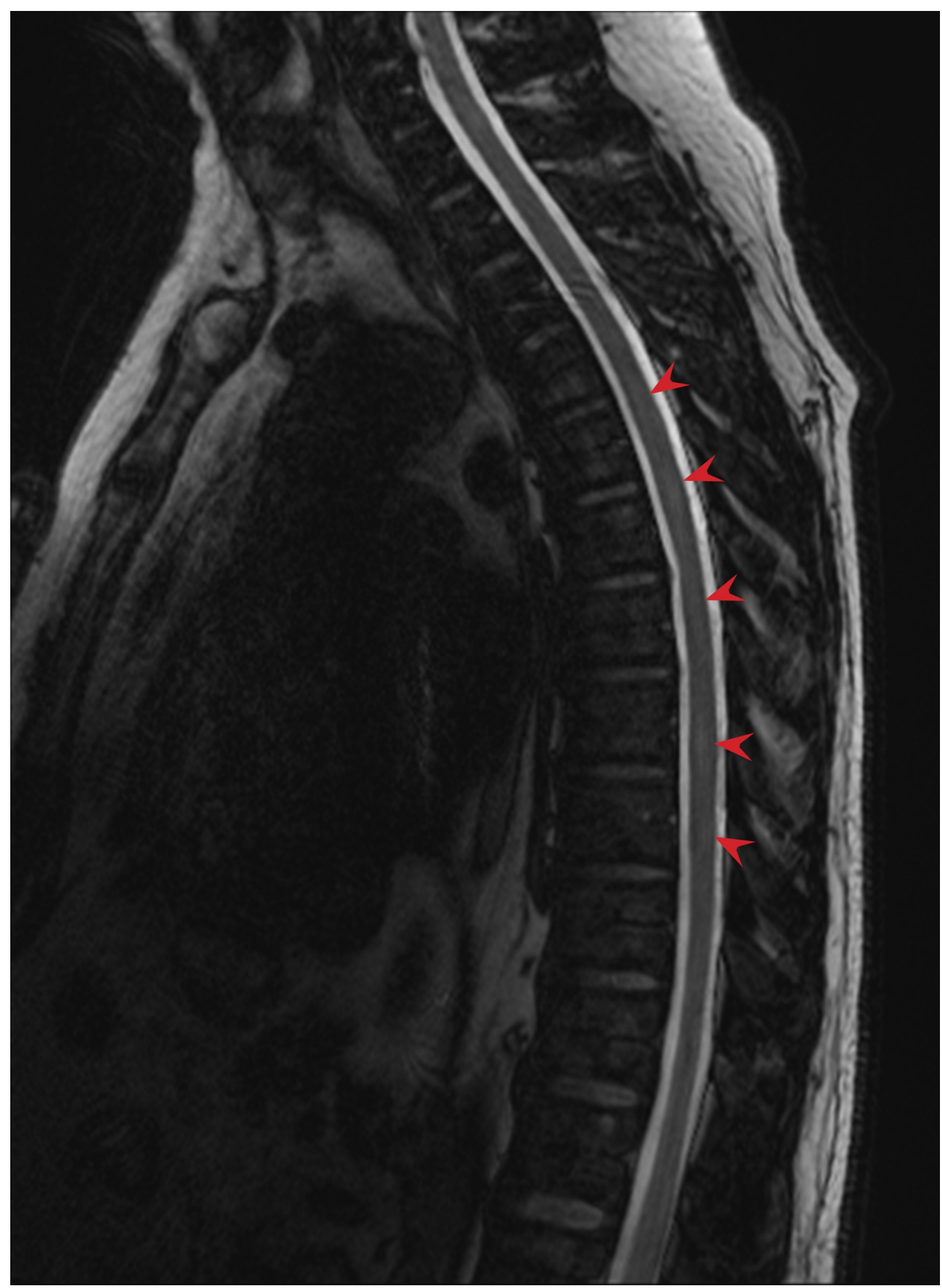

FIGURE 2: Indeterminate signal abnormality at multiple levels (arrows) of the thoracic and lumbar spine on T2-weighted, Dixon water-fat opposed MRI. 


\section{Cureus}

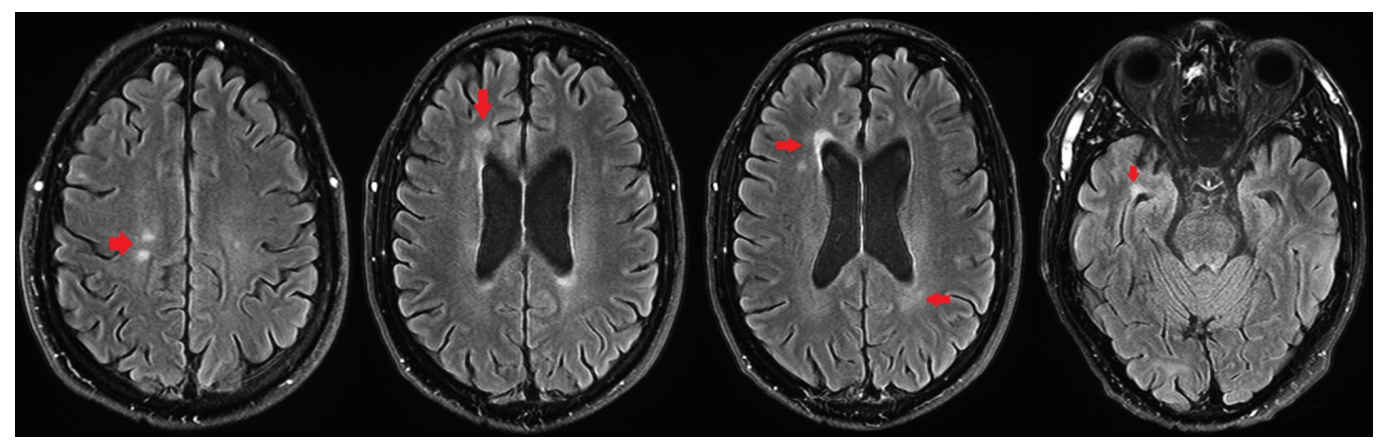

FIGURE 3: Axial fluid-attenuated inversion recovery (FLAIR) T2weighted, fat-suppressed brain MRI demonstrating multiple foci of juxta-ventricular signal hyperintensity (arrows).

Although the diagnosis of MS provided a satisfactory explanation for the patient's otherwise confounding symptoms, he remained with a demonstrably symptomatic cervical schwannoma, now complicated by the possible need for future immunosuppression. Correspondingly, the tumor was approached via a right posterior C6-C7 facetectomy; a gross total resection of the tumor was achieved; and a C6-T1 posterior instrumented fusion was completed, due to concern for possible destabilization in the setting of total facetectomy at a spinal level proximal to the cervicothoracic junction. Pathology confirmed a schwannoma. By post-operative day one, his right upper extremity weakness and radiculopathy were dramatically improved and had resolved by first neurosurgical follow-up at six weeks. The neurology service determined that the extent of his MS disease burden was such that initial management could be expectant; correspondingly, immunosuppression was not recommended and his other symptoms remained stable as of the last neurosurgical follow-up at one year.

\section{Discussion}

Although both spinal schwannomas and MS are relatively common diagnoses, their synchronous presentation is exceedingly rare, with ours marking only the third reported case. Moreover, our review of the literature did not find report of other demyelinating conditions such as neuromyelitis optica or transverse myelitis presenting synchronously with schwannoma, or MS coexisting with an extraspinal schwannoma. Schwannomas are benign neoplasms arising from the myelinating Schwann cell found throughout the peripheral nervous system, with a strong predilection for the cerebellopontine angle and the spinal nerve roots. When arising from spinal nerves, radiculopathy is the typical presentation, beginning with dermatomal pain, which may evolve into progressive focal motor weakness as the tumor grows and mass effect increases [10]. MRI findings include a characteristically well-circumscribed, contrast-enhancing mass, with T2 hyperintensity, and frequent involvement of the neural foramen at the level of disease [2]. More than $95 \%$ of spinal schwannomas occur sporadically; however, several distinct syndromic presentations are frequently observed

[11]. Schwannomatosis is a recently described syndrome defined by multiple schwannomas without presence of a vestibular tumor, and although the overall incidence is rare $(0.58$ cases per one million), spinal involvement is highly prevalent among patients, with three in four harboring at least one spinal nerve root tumor [12]. In parallel, patients with the well-described neurocutaneous phakomatosis neurofibromatosis type two (NF2) very frequently develop spinal schwannomas, accounting for $1-2 \%$ of all such tumors $[1,11]$. For both the sporadic and the syndromic diagnoses, the most common initial symptoms are localized pain, paresthesia/numbness, and motor weakness. Standard-of-care is almost universally surgical resection, although minimally symptomatic lesions can be safely observed, and radiotherapy 
may be considered for patients who are poor surgical candidates.

MS is a truly protean disease, with common presenting symptoms spanning the breadth of neurologic dysfunction, including visual loss, diplopia, sensory disturbance, focal motor weakness, bowel/bladder dysfunction, ataxia, and other cranial neuropathies

[13]. Correspondingly, effectively every conceivable neurologic symptom has been described in MS, including the combination of dermatomal pain, motor weakness, and hyporeflexia that are characteristic of a spinal schwannoma [14]. Given the high degree of variability in clinical, radiographic, and laboratory findings associated with MS, diagnosis is made using the McDonald Criteria, which require dissemination of lesions in time and space [15]. In the modern era, MRI has become sufficiently advanced to date multiple lesions as major timepoints-for example, contrast enhancement is visualized at a different point in the natural history of an MS plaque than non-enhancing T2 hyperintensity, and so simultaneous enhancing and T2-bright lesions are radiographically "separated in time." However, as with the disease's clinical manifestations, the imaging findings are not universally pathognomonic, and MS lesions have been described with radiographic appearances ranging from a spinal cord or nerve root tumor to CNS lymphoma, glioblastoma, metabolic derangements, and a host of other neurologic disorders [16-17]. Consequently, MS is frequently upheld as one of the great pathologic imitators-although it is far more commonplace to find MS in lieu of another diagnosis it was imitating than in addition to it [18].

With these disease features in mind, we turn to the two previously reported cases of concomitant presenting spinal schwannoma and MS. In the index case, Salvi et al. described a 32-year-old man with progressive pain and weakness of the left lower limb, which eventually evolved to encompass the right lower extremity, with simultaneous urinary urgency [19]. MRI of the brain demonstrated multiple white matter lesions concerning for demyelinating disease, while T-spine imaging identified a vividly contrast-enhancing intra-dural extramedullary lesion at T7, which was subsequently resected and confirmed as a spinal schwannoma. Subsequently, Etus et al. described a 46-year-old woman with new right lower extremity paresthesias and urinary incontinence, as well as a two-year history of MS [20]. Physical examination revealed sensory disturbance in a $\mathrm{T} 9$ sensory distribution with bilateral deep tendon hyperreflexia. When these symptoms did not improve with corticosteroid therapy, MRI of the spine was performed, and an intra-dural extramedullary contrast-enhancing mass was identified on the right-sided T10 nerve root, which was surgically resected and diagnosed as a schwannoma. Although the diagnosis of MS was already established in this case, it provides another important illustration of the key clinical principle: that the simplest answer should not universally be assumed as correct, and, from a practical perspective, repeat MR imaging is requisite before a new symptom in a patient with MS can definitively be considered an expression of that primary diagnosis.

\section{Conclusions}

In many clinical circumstances, Osler's Rule proves a still-relevant diagnostic principleparticularly with respect to neurologic diagnoses such as MS, which have the capacity to elicit simultaneous symptoms across a range of anatomically disparate domains, from sensation to locomotion, vision to micturition. Notwithstanding, although a single pathology is undoubtedly the "simpler" and more gratifying explanation to a diagnostic dilemma, clinicians are obliged to weigh all the evidence and to seek out a solution or set of solutions that incorporates all the pertinent history, exam, and imaging findings. As such, our report is not only the third case of synchronously presenting MS and spinal schwannoma, but a compelling reminder of the capacity for atypical cases to confound, and one which emphasizes the importance of bringing fresh eyes and an unbiased perspective to every neurosurgical referral.

\section{Additional Information}




\section{Disclosures}

Human subjects: Consent was obtained by all participants in this study. Conflicts of interest: In compliance with the ICMJE uniform disclosure form, all authors declare the following: Payment/services info: All authors have declared that no financial support was received from any organization for the submitted work. Financial relationships: All authors have declared that they have no financial relationships at present or within the previous three years with any organizations that might have an interest in the submitted work. Other relationships: All authors have declared that there are no other relationships or activities that could appear to have influenced the submitted work.

\section{References}

1. Seppälä MT, Haltia MJ, Sankila RJ, Jääskeläinen JE, Heiskanen O: Long-term outcome after removal of spinal schwannoma: a clinicopathological study of 187 cases. J Neurosurg. 1995, 83:621-626. 10.3171/jns.1995.83.4.0621

2. Sun I, Pamir MN: Non-syndromic spinal schwannomas: a novel classification. Front Neurol. 2017, 8:318. 10.3389/fneur.2017.00318

3. Jinnai T, Hoshimaru M, Koyama T: Clinical characteristics of spinal nerve sheath tumors: analysis of 149 cases. Neurosurgery. 2005, 56:510-515. 10.1227/01.NEU.0000153752.59565.BB

4. Alonso A, Hernán MA: Temporal trends in the incidence of multiple sclerosis: a systematic review. Neurology. 2008, 71:129-135. 10.1212/01.wnl.0000316802.35974.34

5. Ramagopalan SV, Sadovnick AD: Epidemiology of multiple sclerosis . Neurol Clin. 2011, 29:207-217. 10.1016/j.ncl.2010.12.010

6. Braverman DL, Lachmann EA, Tunkel R, Nagler W: Multiple sclerosis presenting as a spinal cord tumor. Arch Phys Med Rehabil. 1997, 78:1274-1276. 10.1016/S0003-9993(97)90344-0

7. Awang SM, Saiful NM, Madhavan M, Abdullah J, Tharakan JK: Demyelinating disease masquerading as a surgical problem: a case series. J Med Case Rep. 2009, 3:7407. 10.4076/1752-1947-3-7407

8. Maloney WJ: Occam's Razor and Hickam's Dictum: the transformation of a theoretical discussion into a modern and revolutionary tool in oral diagnostics. WebmedCentral DENTISTRY. 2011, 2:WMC001914. Accessed: April 20, 2018:

10.9754/journal.wmc.2011.001914

9. Miller WT: Letter from the editor: Occam versus Hickam. Semin Roentgenol. 1998, 33:213. 10.1016/S0037-198X(98)80001-1

10. Li P, Zhao F, Zhang J, et al.: Clinical features of spinal schwannomas in 65 patients with schwannomatosis compared with 831 with solitary schwannomas and 102 with neurofibromatosis type 2: a retrospective study at a single institution. J Neurosurg Spine. 2016, 24:145-154.

11. Gonzalvo A, Fowler A, Cook RJ, Little NS, Wheeler H, McDonald KL, Biggs MT: Schwannomatosis, sporadic schwannomatosis, and familial schwannomatosis: a surgical series with long-term follow-up. J Neurosurg. 2011, 114:756-762. 10.3171/2010.8.JNS091900

12. Merker VL, Esparza S, Smith MJ, Stemmer-Rachamimov A, Plotkin SR: Clinical features of schwannomatosis: a retrospective analysis of 87 patients. Oncologist. 2012, 17:1317-1322. 10.1634/theoncologist.2012-0162

13. Brownlee WJ, Hardy TA, Fazekas F, Miller DH: Diagnosis of multiple sclerosis: progress and challenges. Lancet. 2017, 389:1336-1346. 10.1016/S0140-6736(16)30959-X

14. Clifford DB, Trotter JL: Pain in multiple sclerosis . Arch Neurol. 1984, 41:1270-1272. 10.1001/archneur.1984.04050230052017

15. Thompson AJ, Banwell BL, Barkhof F, et al.: Diagnosis of multiple sclerosis: 2017 revisions of the McDonald Criteria. Lancet Neurol. 2017, 17:162-173. 10.1016/S1474-4422(17)30470-2

16. Kalanie H, Harandi AA, Bakhshandehpour R, Heidari D: Multiple large tumefactive MS plaques in a young man: a diagnostic enigma and therapeutic challenge. Case Rep Radiol. 2012, 2012:1:5. 10.1155/2012/363705

17. Naeem SB, Niazi F, Baig A, Sadiq H, Sattar M: Primary CNS lymphoma vs. tumefactive multiple sclerosis: a diagnostic challenge. J Coll Physicians Surg Pak. 2018, 28:66-68.

10.29271/jcpsp.2018.01.66 


\section{Cureus}

18. Kappos L, Edan G, Freedman MS, et al.: The 11-year long-term follow-up study from the randomized BENEFIT CIS trial. Neurology. 2016, 87:978-987.

10.1212/WNL.0000000000003078

19. Salvi F, Mascalchi M, Plasmati R, Michelucci R, Calbucci F, Dal Pozzo G, Tassinari C: Multiple lesions in cerebral white matter in two young adults with thoracic extramedullary tumours. J Neurol Neurosurg Psychiatry. 1992, 55:216-218. 10.1136/jnnp.55.3.216

20. Etus V, Akansel G, Ilbay K, Koç K, Ceylan S: Multiple sclerosis and coexisting intradural extramedullary spinal cord tumour: a case report. Neurol Sci. 2002, 23:119-122.

$10.1007 / \mathrm{s} 100720200037$ 